\title{
Metastatic cervical lymph nodes: general practitioner referral patterns
}

\author{
Duncan McRae ${ }^{1}$, George Buchanan ${ }^{2}$ and G.S. Kenyon ${ }^{1}$ \\ ${ }^{1}$ The Royal London Hospital, Whitechapel, London E1 1BB, and ${ }^{2}$ Southend Hospital, Prittlewell Chase, \\ Westcliff-on-Sea, Essex SSO ORY, UK
}

\begin{abstract}
Summary: Premature excision biopsy of a cervical lymph node infiltrated by metastatic carcinoma may compromise patient survival since it is associated with an increased incidence of local wound recurrence and distant metastases. Seventy per cent of such patients have an identifiable head and neck primary, obviating the need for an excision biopsy. It is important therefore that they are examined by surgeons who are experienced in inspecting the upper aerodigestive tract and who are competent in performing definitive head and neck surgery. A questionnaire sent to all general practitioners of an Inner London and a District Health Authority revealed that only $18 \%$ and $33 \%$ respectively referred patients who they suspected of having a metastatic neck node to a department with an experienced head and neck surgeon. We conclude that greater emphasis on the correct management of these patients at both an undergraduate and postgraduate level may encourage subsequent generations of general practitioners to review their referral patterns.
\end{abstract}

\section{Introduction}

The asymptomatic enlargement of one or more cervical lymph nodes in an adult has at least an $85 \%$ chance of being malignant. ${ }^{1}$ Seventy per cent of malignant cervical nodes are metastases from primary head and neck cancers. ${ }^{2}$ The majority of the remainder are due to lymphomas. Factors suggesting malignancy include node size greater than $1.5 \mathrm{~cm}$ in diameter, hard painless nodes and rapid enlargement. ${ }^{3}$

The correct management of patients suspected of having metastatic cervical lymph nodes is well documented. ${ }^{4} \mathrm{~A}$ thorough search for a head and neck must be made and if an experienced cytologist is available a fine needle aspiration biopsy of the neck primary lump should be performed before resorting to an excision biopsy. Premature excision biopsy of a metastatic neck node will increase the incidence of distant metastases and may cause local wound complications such as tumour recurrence, fungation and infection. ${ }^{5,6}$ It may therefore compromise patient survival. Furthermore an inappropriately placed incision can upset the planning of a future radical neck dissection. ${ }^{5}$ For these reasons referral to a surgeon experienced in examining the upper aerodigestive tract and in performing definitive neck surgery is desirable.

Correspondence: D. McRae, F.R.C.S.

Accepted: 18 November 1991
Head and neck surgeons well known to the loca general practitioners (GPs) practise at The Royatô London and Southend Hospitals, the Ear, Nose and Throat (ENT) centres of those Health Authorities. It was the authors' impression, however, that many of the patients seen with a metastatic neck node were internal hospital referrals, the patients in many instances having already had a premature excision biopsy without prior upper aerodigestive tract assessment. One possible source of this error was the referral pattern of the local GPs. To investigate this impression and, if proven, to establish the reasons for it, a questionnaire was sent to all GPs within the London Borough of Tower Hamlets and Southend Health Authority.

\section{Materials and methods}

General practitioners registered with the Family Practitioner Committees of both Health Authorities were asked to consider the following scenario:

A previously fit 40 year old Caucasian male presents to you with a hard, painless, mobile 3 by $3 \mathrm{~cm}$ deep mid-cervical neck lump. This was first noticed 2 months previously and is increasing in size. You strongly suspect the lump to be a lymph node infiltrated by metastatic carcinoma. You can detect no symptoms or signs of a primary carcinoma. 
They were asked to state their referral preference and to comment on the reasons behind their mode of referral.

\section{Results}

Replies were received from a total of 153 out of 181 GPs circularized in the Southend area, a response rate of $84.5 \%$. The Tower Hamlets equivalent figures were 76 replies from 94 , a response rate of $80.9 \%$. Not all respondents gave a reason for their mode of referral.

The results of the questionnare are shown in Tables I and II. It is immediately clear that the preferred route of referral for a patient with a potentially malignant cervical lymph node is to a general surgeon. This finding is true for both regions surveyed (53.9\% Tower Hamlets and $48.4 \%$ Southend). The reasons given for this were to obtain an immediate tissue diagnosis $\mathbf{4 5 . 9 \%}$ Southend and $31.7 \%$ Tower Hamlets of those GPs referring to general surgeons) and the speed in which urgent cases are seen $(41.5 \%$ Tower Hamlets and $17.1 \%$ Southend of the same GP group).

There were also those respondents in both areas who expressed the belief that the primary was likely to arise outside the head and neck or who felt a better work-up would be obtained by an alternative

Table I Referral mode of preference

\begin{tabular}{lcc}
\hline Referral preference & Southend & Tower Hamlets \\
\hline General surgeons & $74(48.4 \%)$ & $41(53.9 \%)$ \\
ENT surgeons & $50(32.7 \%)$ & $14(18.4 \%)$ \\
General physicians & $11(7.2 \%)$ & $10(13.2 \%)$ \\
Oncologists & $5(3.3 \%)$ & $4(5.3 \%)$ \\
No preference between & $5(3.3 \%)$ & $4(5.3 \%)$ \\
$\quad$ ENT or general surgeons & & \\
Dependent on GPs own & $4(2.6 \%)$ & $2(2.6 \%)$ \\
$\quad$ initial investigations & & \\
Phone for advice & $2(1.3 \%)$ & $1(1.3 \%)$ \\
Plastic surgeons & $1(0.7 \%)$ & 0 \\
Chest physicians & $1(0.7 \%)$ & 0 \\
\hline
\end{tabular}

referral. For these reasons a significant number of referrals in both regions surveyed were preferentially made to general surgeons, oncologists and general physicians.

There was a group of GPs who recognized that patients with a metastatic mid-cervical neck node most likely had an upper aerodigestive tract primary and referred appropriately to a department with an experienced head and neck surgeon (36 $(23.5 \%)$ GPs in Southend and eight GPs $(10.5 \%)$ in Tower Hamlets).

\section{Discussion}

An enlarging, painless, hard cervical neck lump in an adult should be considered to be a metastasis from a primary malignancy of the upper aerodigestive tract unless proved otherwise.

Lindberg in reviewing 2,044 patients with head and neck cancer found the commonest primary sites of cervical lymph node metastasis to be the nasopharynx, oropharynx, tongue base, hypopharynx, supraglottic larynx and tonsil ${ }^{8}$ (Figure 1). He emphasized, however, that supraclavicular as opposed to cervical metastatic deposits arise from primary tumours below the head and neck, principally the bronchus and stomach. His findings correlate with other published data. ${ }^{9-13}$ Initial assessment should therefore include indirect mirror or fibreoptic rhinolaryngoscopic examination of the upper aerodigestive tract. Of those primary malignancies encountered, more than $70 \%$ will be identified on otorhinolaryngological assessment. ${ }^{14}$

Further investigations, including head and neck radiological examination and an upper aerodigestive tract panendoscopy under general anaesthesia with free field biopsy of the post-nasal space are indicated when a primary malignancy is not identified on initial assessment. A positive biopsy from a primary head and neck malignancy obviates the need for an excision biopsy of the cervical neck node. The neck node may then be treated definitively by including it in a radical neck dissection or in a radiotherapy field.

Table II Reasons for referral

\begin{tabular}{|c|c|c|}
\hline Reason & Southend & Tower Hamlets \\
\hline $\begin{array}{l}\text { The primary is likely to be in the upper } \\
\text { aerodigestive tract }\end{array}$ & 36 (all referred to ENT) & 8 (all referred to ENT) \\
\hline To get an initial histological diagnosis & $\begin{array}{l}34 \text { (to general surgeons) } \\
2 \text { (to ENT) }\end{array}$ & $\begin{array}{l}13 \text { (to general surgeons) } \\
1 \text { (to ENT) }\end{array}$ \\
\hline $\begin{array}{l}\text { The speed with which urgent cases } \\
\text { are seen }\end{array}$ & 13 (to general surgeons) & 17 (to general surgeons) \\
\hline $\begin{array}{l}\text { The primary is likely to be outside the } \\
\text { upper aerodigestive tract }\end{array}$ & $\begin{array}{l}3 \text { (to general surgeons) } \\
1 \text { (to oncology) }\end{array}$ & $\begin{array}{l}7 \text { (to general surgeons) } \\
2 \text { (to oncology) }\end{array}$ \\
\hline Better work up to find the primary & 6 (to general physicians) & 3 (to general physicians) \\
\hline
\end{tabular}




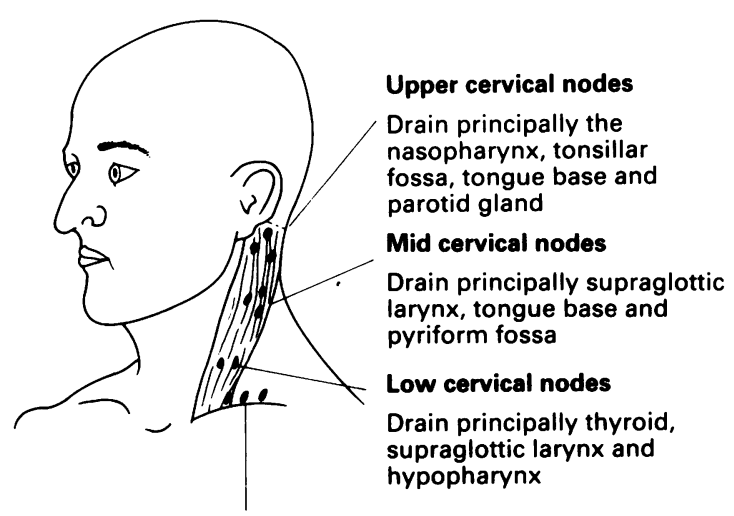

Supraclavicular nodes

Drain principally bronchus, breast, cervical oesophagus and stomach

Figure 1 Cervical and supraglottic node location and their principal drainage areas (after Lindberg).

If an occult primary is encountered and if an experienced cytologist is available then fine needle aspiration biopsy of a neck node may allow for definitive treatment of a metastatic node without the need for an excision biopsy., ${ }^{6,15,16}$ It is a highly sensitive and specific investigation for squamous metastasis ${ }^{6}$ but is much less accurate for other tumour types. ${ }^{15,16}$

Only when an occult primary with negative or equivocal fine needle aspiration cytology of the neck node is encountered is an excision biopsy indicated. This should be performed by an experienced head and neck surgical team to ensure that the oral cavity, pharynx and larynx are free of disease and who can place the incision so it may be included in a planned approach for a radical neck dissection: this may be performed immediately should positive frozen section histology be obtained. ${ }^{17}$

The ENT and General Surgery departments at

\section{References}

1. Jaques, D.A. Management of metastatic nodes in the neck from an unknown primary. In: Paperella, M.M. \& Shumrick, D.A. (eds) Otolaryngology. W.B. Saunders, Philadelphia, 1980, pp. 2998-3003.

2. Stell, P.M. Management of malignant nodes of the neck. In: Bull, T.R., Ransome, J. \& Holden, H.B. (eds) Recent Advances in Otolaryngology. Churchill Livingstone, London, 1978, pp. 125-139.

3. Gregos, R.T., Waner, M., Davidge-Pitts, K.J. \& Joseph, L.A. Evaluation of suspected malignant lymph nodes in the neck. $S$ Afr J Surg 1985, 23: 79-82.

4. Premachandra, D.J., McRae, D. \& Prinsley, P. Biopsy of neck lumps in adults should be preceded by examination of the upper aerodigestive tract. Postgrad Med J 1990, 66: 113-115.
Southend and The Royal London Hospitals see urgent referrals within a fortnight. Routine referrals are seen much more rapidly by the general surgeons at both hospitals. This perceived delay to consultation may underlie this reason being given for the preferred referral to general surgeons.

It is disturbing to find some GPs thought a mid deep cervical metastatic neck node most likely arose from a primary outside the upper aerodigestive tract $(9.2 \%$ Tower Hamlets and $2.0 \%$ Southend) or that a general physician would provide the best opinion for such a patient. When an appropriate referral was made, it was usually because of the realization that the most likely site of the primary was the head and neck ( 36 out of 50 (or $72 \%$ ) of Southend GPs and eight out of 14 (or $57.2 \%$ ) of Tower Hamlets GPs referring to a head and neck surgeon). The overall number of referrals to a surgeon experienced in the management of patients with head and neck cancer is very low in both regions surveyed $(32.7 \%$ Southend and $18.4 \%$ Tower Hamlets) which confirms the clinical suspicion that many such patients are being inappropriately referred.

The latest published stastistics show that 2,800 develop and 1,720 die of upper aerodigestive tract cancer each year in England and Wales. ${ }^{18,19}$ This dismal prognosis can only be improved by primar's referral to a surgeon experienced in diagnosing an managing head and neck cancer. The greatef percentage of referrals to a head and neck surgeon? from the Southend group of general practitioners may reflect a greater involvement of the local ENT surgeons in postgraduate education. This implies a way forward. We suggest there is a need, at both an undergraduate and postgraduate level, for greater emphasis on teaching the management of an adult presenting with an enlarging neck node.

No surgeon would excise an axillary lymph node without first examining the breast. Similarly no surgeon should excise a cervical lymph node unless the upper aerodigestive tract has been examined by a head and neck surgeon. ${ }^{4}$

5. McGuirt, W.F. \& McCabe, B.F. Significance of node biopsy before definitive treatment of cervical metastatic carcinoma Laryngoscope 1978, 88: 594-597.

6. Birchall, M.A., Walsh-Waring, G.P. \& Stafford, N.D. Malignant neck lumps: a measured approach. Ann $R$ Coll Surg Engl 1991, 73: 91-95.

7. Damion, J. \& Hybles, R.L. The neck masses. Postgrad Med 1987, 81: 97-107.

8. Lindberg, R. Distribution of cervical lymph node metastases from squamous carcinoma of the upper respiratory and digestive tracts. Cancer 1972, 291: 1446-1500.

9. Marchetta, F.C., Murphy, W.T. \& Kovaric, J.J. Carcinoma of the neck. Am J Surg 1963, 974: 974-979. 
10. Aquarelli, M.J., Matsunga, R.S. \& Cruze, K. Metastatic carcinoma of the neck of unknown primary origin. Laryngoscope 1961, 71: 962-974.

11. Ridenhour, C.E., Yeun, P. \& Sprat, J.S. Metastatic carcinoma in cervical lymph nodes from occult primary sites. Missouri Med 1967, 64: 988-993.

12. Pico, J., Frias, Z. \& Bosch, A. Cervical lymph node metastasis from carcinoma of undetermined origin. $A J R$ 1971, 111: 95-102.

13. Barrie, J.R., Knapper, W.H. \& Strong, E.W. Cervical lymph node metastasis of unknown origin. Am J Surg 1970, 120: 466-470.

14. Miller, S.M., Hamaker, R.C. \& Singer, M.I. The metastatic malignant neck mass. Indiana Med 1986, 79: 516-518.
15. Frable, W.J. \& Frable, M.A. Thin needle aspiration biopsy: the diagnosis of head and neck tumours revisited. Cancer 1979, 43: 541-548.

16. Young, J.E.M., Archibald, S.D. \& Shier, K.J. Needle aspiration cytologic biopsy in head and neck masses. Am J Surg 1981, 142: 484-489.

17. Martin, H. Untimely lymph node biopsy. Am J Surg 1961, 102: $17-18$.

18. Cancer statistics registrations: England and Wales - 1985; Office of Population Censuses and Surveys, 1990; HMSO, Series MB1 no. 18.

19. Cancer mortality statistics: England and Wales - 1989; Office of Population Censuses and Surveys, 1990; HMSO, Series DH2 no. 16. 